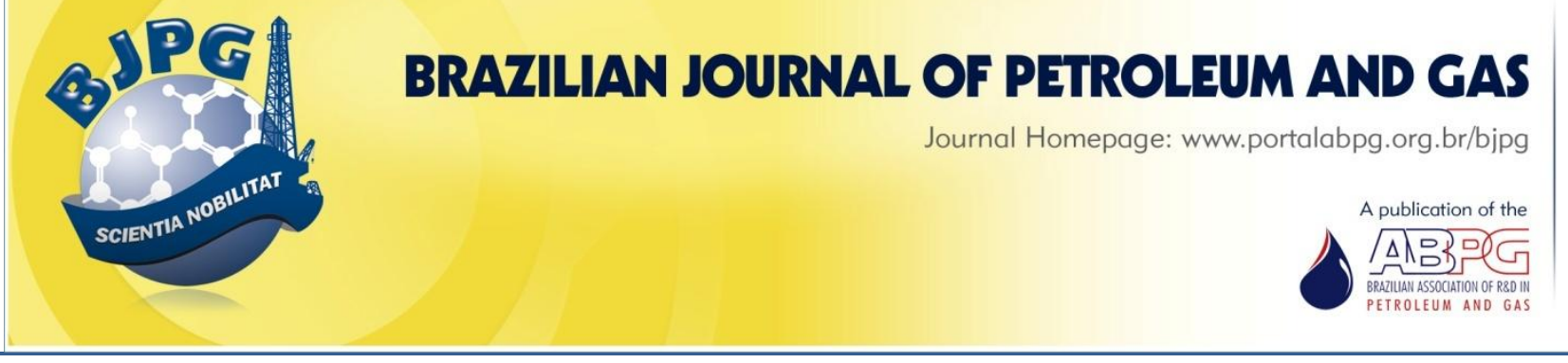

\title{
IMPACT OF NUMBER OF FRACTURES ON OIL RECOVERY DURING ELECTROMAGNETIC HEATING
}

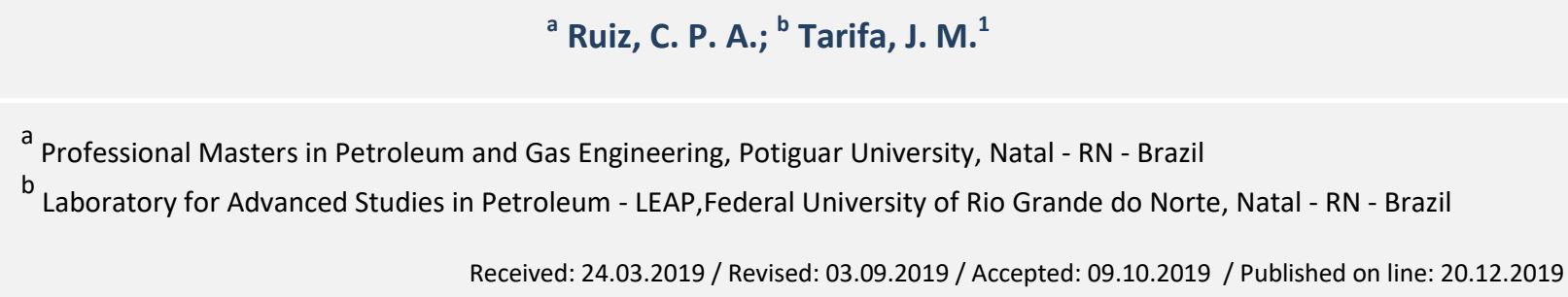

\begin{abstract}
Thermal recovery methods are used typically to produce heavy oil and bitumen. During this process, the temperature is raised to reduce oil viscosity. Conventional thermal methods may not be effective on deep wells or thin zones. An alternative to the thermal process is electromagnetic heating, which is a process based on the transformation of electric energy into thermal energy due to the electromagnetic field and electrically sensitive particles interacting on the medium. In this paper, reservoir simulations with similar characteristics to those found in the sedimentary basins of the Brazilian Northeast were performed. The purpose of this work is to analyze the impact of the number of fractures, fracture configurations and number of producer wells during electromagnetic heating over the oil recovery factor (ORF) value. The results revealed an oil production increment with increasing number of producer wells and when fractures are located at the center of the oil production zone. However, oil production did not show an evident increase connected to the number fractures.
\end{abstract}

\section{KEYWORDS}

electromagnetic heating; reservoir simulation; enhanced oil recovery; cracking reactions

\footnotetext{
${ }^{1}$ To whom all correspondence should be addressed.

Address: Laboratory for Advanced Studies in Petroleum - LEAP, Federal University of Rio Grande do Norte - UFRN, Campus Universitário Lagoa Nova, Natal/RN/Brazil.

ZIP Code: 59078-900 | Phone number: +55 84 99158-3382 | e-mail: jhonmt@ufrnet.br

doi:10.5419/bjpg2019-0020
} 


\section{INTRODUCTION}

Developments in technology and industry seek to increase the production of unconventional hydrocarbons from heavy oil reservoirs. Due to its high viscosity, heavy oil became very expensive and challenging to produce. That is why steam and hot water injection and in-situ combustion are the most common techniques used to overcome this problem. However, these thermal processes are controlled poorly because their efficiency is affected easily by aspects such as reservoir heterogeneity, thickness, depth, and oil saturation. Moreover, these traditional thermal methods are costly and environmentally harmful.

Electromagnetic Heating (EMH) can be separated into three categories depending on the frequency of the electrical current: resistive or low frequency heating, induction heating, and high frequency heating.

Low frequency heating is also known as resistive heating. During this process, the electric current passes through a formation until it reaches conductive materials which produce heat and transmit it to the reservoir (Da Mata, 1993; Peraser et al., 2012).

The inductive electromagnetic heating applies to projects that use a specific configuration of electric conductors that create a magnetic field to generate an electric current (Koolman et al., 2008; Mustafina et al., 2013).

In high frequency heating, the energy of the electromagnetic waves is transmitted to a medium (connate water, in this case). The electric field changes its direction and the water dipole must rotate (molecules rotation generates heat) to maintain the same direction with the polarity. Evaporation of connate water creates a vapor chamber that grows progressively with heat and time. To accelerate the oil production, it is suggested to use and additional displacement mechanism, such as gas injection at the top of the reservoir (Sahni et al., 2000; Oliveira et al., 2009; Gasbarri et al., 2011).

This research aims to analyze the impact of the number of fractures, the fractured layer position, and the possibility of using one or two producer wells during electromagnetic heating.

\section{RESERVOIR MODELING AND METHODOLOGY}

The present study used data values based on the information (reservoir properties) given on $\mathrm{PhD}$ Thesis of Dr. Araújo Júnior (Araújo Júnior, 2015). For modeling and simulating oil and reservoir properties and their behavior, this study used the Computer Modelling Group (CMG) commercial simulator and its corresponding modules.

The characteristics of the physical model proposed for this study are detailed in a previous work (Ruiz et al., 2016) and presented in Table 1.

Other characteristics of the physical model were the total number of blocks $=6,400$, the length $=$ Width $=16$ blocks of 6.5 meters each and the height $=25$ blocks with variable dimensions. The modeled fluid represents heavy oil with initial viscosity of $1246.81 \mathrm{cp}$.

Different models of cracking reactions and their kinetic values were taken from Tarifa et al. (2017). Those models represented a realistic situation

Table 1. Properties of the Reservoir.

\begin{tabular}{ll}
\hline Initial Temperature $\left({ }^{\circ} \mathrm{C}\right)$ & 38 \\
Oil zone height $(\mathrm{m})$ & 20 \\
Porosity $(\%)$ & 28 \\
Porosity of the fractured zone (\%) & 60 \\
Horizontal Permeability $(\mathrm{md})$ & 1,000 \\
Vertical Permeability $(\mathrm{md})$ & 100 \\
Permeability of the fractured zone $(\mathrm{md})$ & 10,000 \\
\hline
\end{tabular}


Table 2. Stoichiometric balance and Kinetics values.

\begin{tabular}{|c|c|c|c|}
\hline \multicolumn{4}{|c|}{ Belgrave et al. Model (1993) modified by the authors } \\
\hline & & $\mathrm{Ar}$ & $\mathrm{Ea}$ \\
\hline R1 & $\mathrm{C}_{32-40+} \rightarrow 11.3163 \mathrm{CO}_{2}$ & $1.18 \mathrm{E}+14$ & 75,796 \\
\hline $\mathrm{R} 2$ & $\mathrm{C}_{32-40+} \rightarrow 38.3014$ Coke & $3.51 E+14$ & 76,183 \\
\hline R3 & $C_{21-31} \rightarrow 0.3502 C_{32-40+}$ & $7.86 \mathrm{E}+17$ & 100,904 \\
\hline R4 & $\mathrm{C}_{13-20} \rightarrow 0.4650 \mathrm{C}_{21-31}$ & $7.86 \mathrm{E}+17$ & 100,904 \\
\hline R5 & $C_{6-12} \rightarrow 0.4301 C_{13-20}$ & $7.86 \mathrm{E}+17$ & 100,904 \\
\hline
\end{tabular}

regarding the formation of coke during the application of a thermal method. The model and the kinetic values used to describe the cracking reactions during the temperature raising correspond to Belgrave's model (Belgrave et al., 1993; Tarifa et al., 2017), and are presented in Table 2.

All fractures analyzed are assumed to have an area of 36 square meters, $6 \mathrm{~m} \times 6 \mathrm{~m}$, located at layers $3,7,12$, and 21 , as shown in Figure 1 . The assumed temperature for all cases is $300^{\circ} \mathrm{C}$.

Figure 1 shows the number of the layers where the fractures were positioned and the number of the production wells. Simulations were performed according to a full factorial design during a period of 20 years, resulting in 30 different combinations of simulations.

\section{RESULTS}

Tarifa et al. (2017) showed that the coke deposition reduces the oil production due to the blockage of the fractured zone and the pore area near to the heated zone. Based on this finding, it was decided to evaluate the number of fractures. The first analysis correlates the influence of the position of one fracture and one production well, Figure 2. The oil recovery factor (ORF) was compared to that obtained by the primary production of one well.

According to Figure 2, fractures at layers 3 and 7 presented almost the same ORF, around $15 \%$. While those fractures located below obtained lower values, ORF around $14 \%$ for the fracture at layer 12 and $9 \%$ for the one located at layer 21 , showing that the lower the fracture's location is the lower the ORF will be. Also, fractures located around the middle of the reservoir help produce oil faster than others less centered, as a larger heated area was achieved through the process.

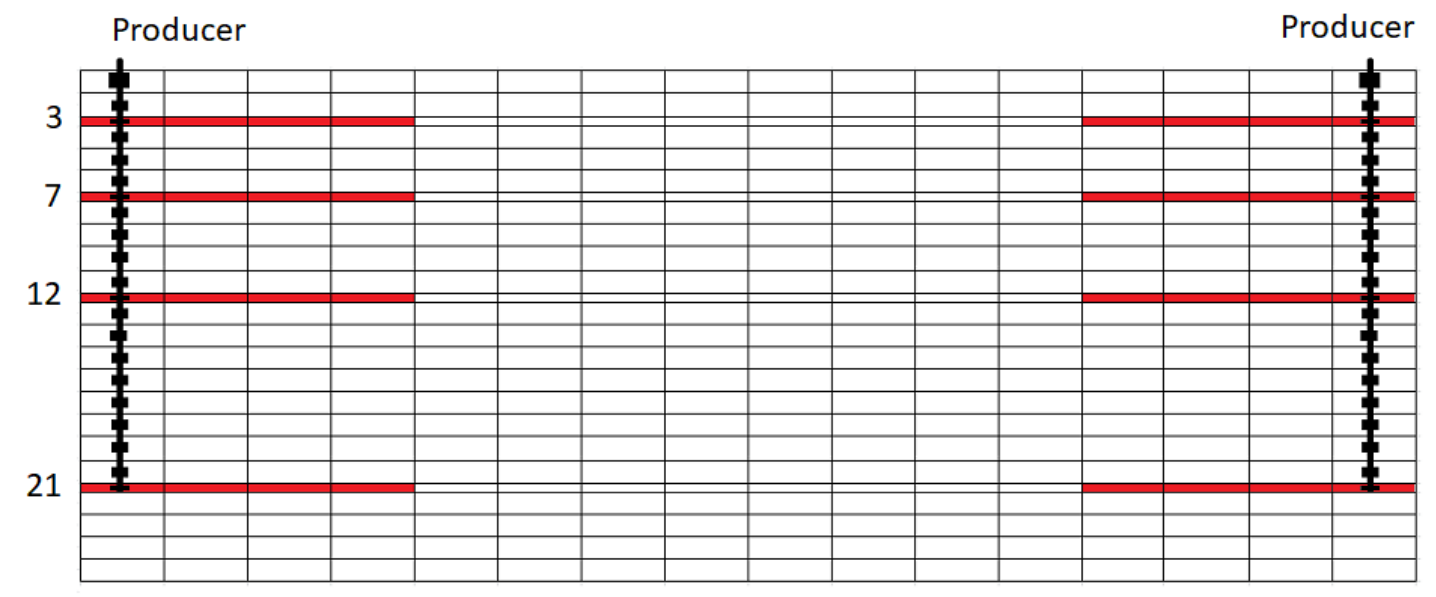

Figure 1. Location of fracture zones. 


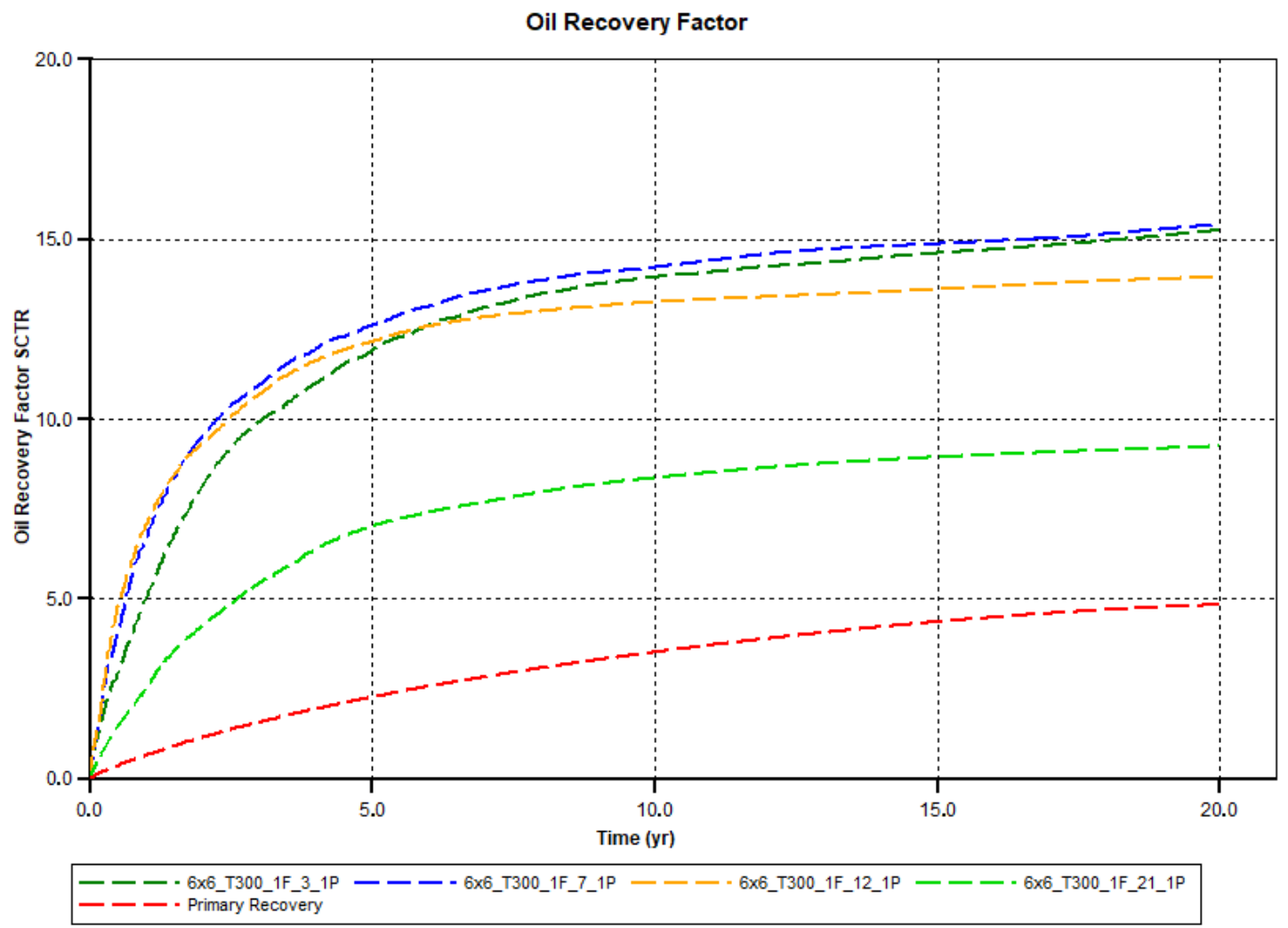

Figure 2. Oil recovery factor for each fractured zone.

Table 3 shows the ORF of the different fractured layer locations and the interaction between them. The higher ORF value was obtained for almost all single fractures rather than with two, three, or four fractures. This result is due to the coke deposition in the fractured zone and its surroundings, reducing the pore volume and, consequently, the permeability. Figure 3 illustrates the coke deposition process. The ORF was lower when the fractured zone was located within the aquifer. Differently, ORF would have increased if other fractures where present within the oil zone.
Figure 3 presents the evolutions of the temperature and the coke deposition when one and three fractures were evaluated.

Figure 3 shows temperature and coke deposition in the fracture zones and their surroundings area for different times. The two first columns, from left to right, show the properties at two and six months, followed by two and twenty years for the model with one fracture. Columns three and four represent the same results for the model with three fractures. As mentioned, a higher number of fractures increases the temperature of a

Table 3. Oil recovery factor for one producer well.

\begin{tabular}{cccccc}
\hline \multicolumn{7}{c}{ ORF for one producer well } \\
\hline Case & ORF & Case & ORF & Case & ORF \\
\hline 1F_3 & 15.25 & 2F_3_12 & 12.85 & 3F_3_7_12 & 11.94 \\
1F_7 & 15.43 & 2F_3_21 & 13.06 & 3F_3_7_21 & 12.31 \\
1F_12 & 13.96 & 2F_7_12 & 12.15 & 3F_3_12_21 & 12.89 \\
1F_21 & 9.25 & 2F_7_21 & 12.72 & 3F_7_12_21 & 11.96 \\
2F_3_7 & 13.25 & 2F_12_21 & 11.22 & 4F_3_7_12_21 & 11.77
\end{tabular}




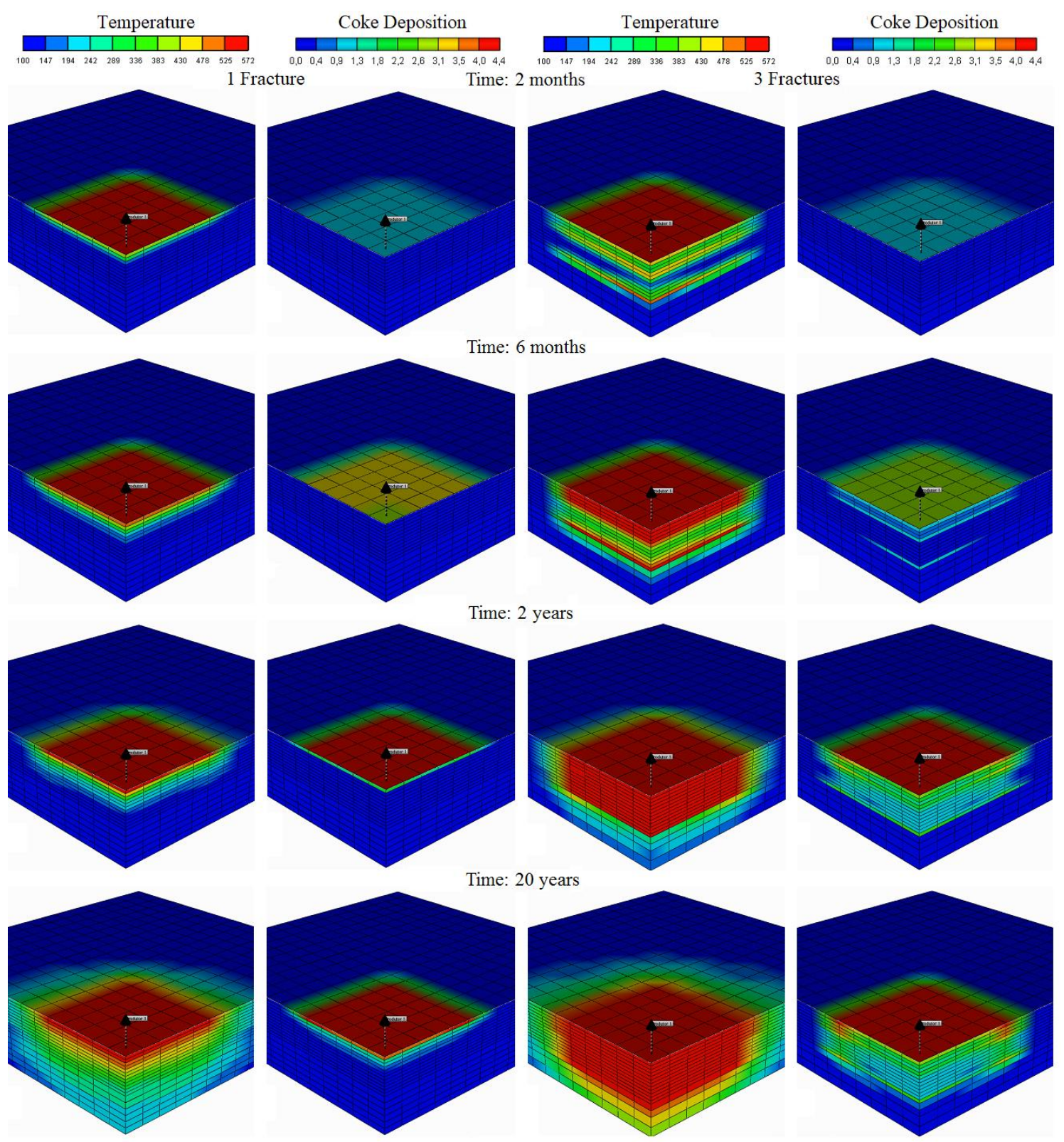

Figure 3. Coke deposition and temperature maps.

larger area, nevertheless, this causes more coke deposition reducing the pore volume and hindering the oil production.

According to Ruiz et al. (2016), each fracture provides a certain temperature because of the electromagnetic heating. It implies that, depending on the number of fractures and their sizes, the temperature around them would rise, generating a larger amount of coke deposition. In this case, one fracture means there was a temperature rise, but less coke deposition when comparing the results between one and four fractures, easing the displacement of oil to the producing wells.
It was observed that for the specific case of a fractured area of $6 \times 6$, and temperature of $300{ }^{\circ} \mathrm{C}$, the best results were presented for cases with one fracture rather than three fractures.

Based on the previous results it was decided to analyze the influence of a second producer well. Figure 4 shows the oil recovery factor of fractures, at different locations, for one and two producer wells.

Figure 4 shows the ORF for the different locations of the fractured zone for cases when one and two producer wells are evaluated. As expected, the ORF for cases with two producer 


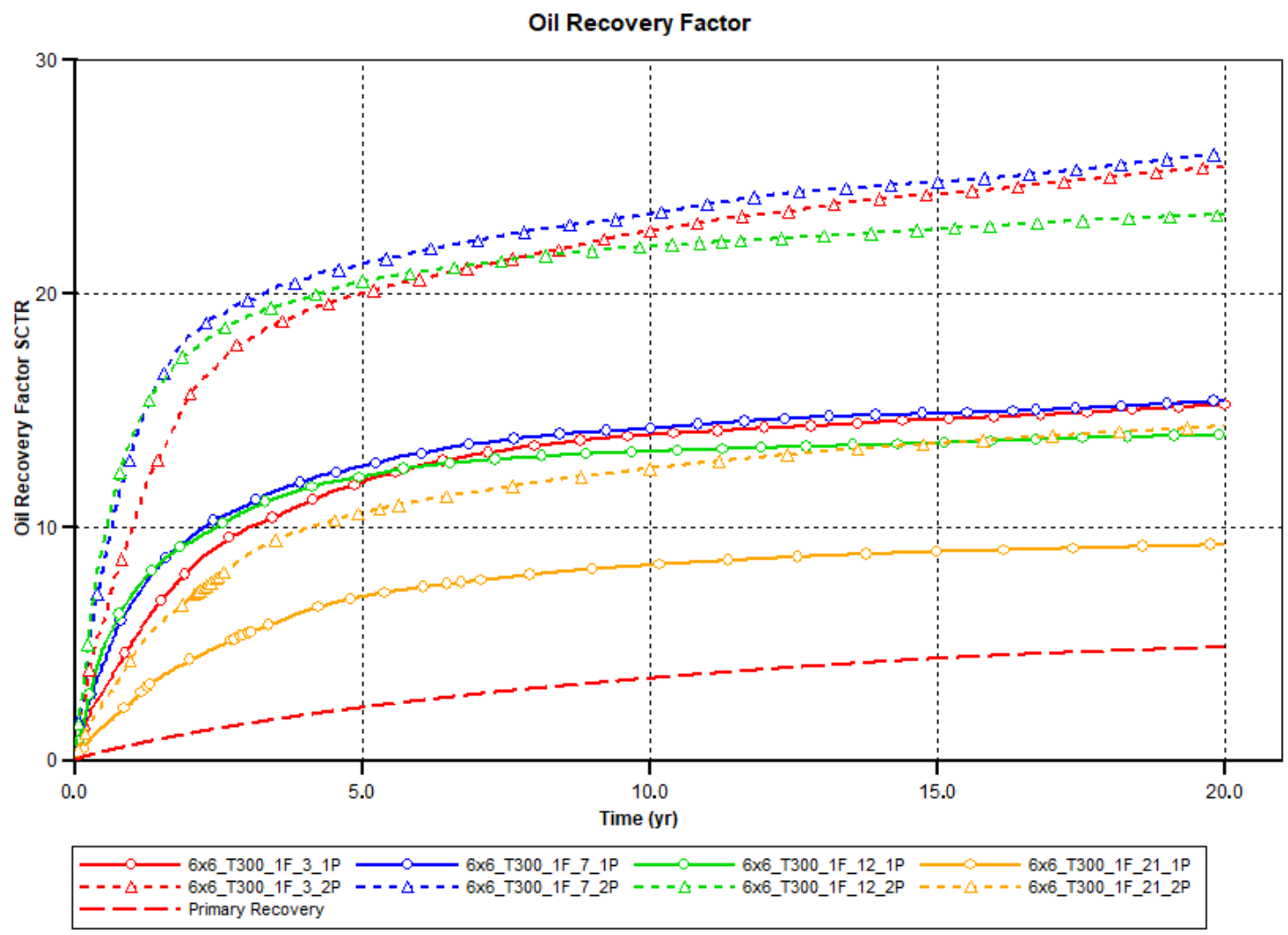

Figure 4. Oil recovery factor for one and two producer wells.

wells is higher than those with a single producer, but the value for two wells is not double than that found for just one, due to the production interference of each well.

Table 3 shows the ORF of the different fractured layer locations when two producer wells are evaluated.

Table 4 shows the ORF for all cases with two producer wells. As previously mentioned, the higher ORF obtained belonged to almost all the case with one fracture rather than with two, three, or four fractures. As in the situation with just one producer, this happens because of the coke deposition in the fractured zone and the surrounding areas. Tables 3 and 4 show that the ORF does not present twice the value when one producer is compared with two producers.

Table 4. Oil recovery factor for two producer wells.

\begin{tabular}{cccccc}
\hline \multicolumn{7}{c}{ ORF for two producer wells } \\
\hline Case & ORF & Case & ORF & Case & ORF \\
\hline 1F_3 & 25.46 & 2F_3_12 & 23.17 & 3F_3_7_12 & 20.93 \\
1F_7 & 26.02 & 2F_3_21 & 22.47 & 3F_3_7_21 & 21.66 \\
1F_12 & 23.38 & 2F_7_12 & 21.23 & 3F_3_12_21 & 22.95 \\
1F_21 & 14.33 & 2F_7_21 & 22.24 & 3F_7_12_21 & 21.13 \\
2F_3_7 & 22.38 & 2F_12_21 & 19.20 & 4F_3_7_12_21 & 20.90 \\
\hline
\end{tabular}




\section{CONCLUSIONS}

The objective of this work was to analyze the impact of the number of fractures, fractured layer position, and number of producer wells during electromagnetic heating.

The situations analyzed showed an increment on the oil production when fractures were located in the center, within the oil zone.

The higher oil recovery factor was obtained with a single fracture rather than with two, three, or four fractures, due to higher coke deposition of the heated zone, reducing oil production due to pore blockage of the fractured zone and the surrounding areas.

The number of wells increased the oil recovery factor, but not linearly.

The coke formation is a critical factor. To avoid over or underestimating the oil recovery factor and represent a realistic situation, laboratory data needs to be collected.

\section{ACKNOWLEDGMENTS}

The authors wish to thank Potiguar University (UnP), PRH-PB 221 (Petrobras), the Computer Modelling Group (CMG), and the Laboratory for Advanced Studies in Petroleum (LEAP-UFRN) for the support provided for the accomplishment of this work.

\section{REFERENCES}

Araújo Júnior, A. D. Recuperação avançada de óleos pesados em poços fraturados utilizando aquecimento eletromagnético indutivo. 153f. Tese de Doutorado. Programa de Pós-Graduaçao em Ciência e Engenharia de Petróleo, Universidade Federal do Rio Grande do Norte, 2015. (in Portuguese)

Belgrave, J. D. M.; Moore, R. G.; Ursenbach, M. G.; Bennion, D. W. A comprehensive approach to in-situ combustion modeling. SPE-20250-PA, SPE Advanced Technology Series, v. 1(1), p. 98-107, 1993. https://doi.org/10.2118/20250-PA

Da Mata, W. Récuperation assistée des petroles visqueux par ondes electromagnétiques. Tese de Doutorado. INPT-ENSEEIHT, Toulouse, França, 1993.
Gasbarri, S.; Diaz, A.; Gusman, M. Evaluation of electric heating on recovery factors in extra heavy oil reservoirs. SPE-149779-MS. SPE Heavy Oil Conference and Exhibition, Kuwait City, Kuwait, 12-14 December, 2011. https://doi.org/10.2118/149779-MS

Koolman, M.; Huber, N.; Diehl, D.; Wacker, B. Electromagnetic heating method to improve steam assisted gravity drainage. SPE-117481-MS. International Thermal Operations and Heavy Oil Symposium, Calgary, Alberta, Canada, 20-23 October, 2008. https://doi.org/10.2118/117481-MS

Mustafina, D.; Kock, A.; Danov, V.; Sotskiy, S. Mechanism of heavy oil recovery driven by electromagnetic inductive heating. SPE-165507MS. SPE Heavy Oil Conference - Canada, Calgary, Alberta, Canada, 11-13 June, 2013.

Oliveira, H. J. M.; Barillas, J. L. M.; Da Mata, W.; Dutra Jr, T. V. Energetic optimization to heavy oil recovery by Electromagnetic Resistive Heating (ERH). SPE-122073-MS. Latin American and Caribbean Petroleum Engineering Conference, Cartagena de Indias, Colombia. 31 May -3 June, 2009. https://doi.org/10.2118/122073-MS

Peraser, V.; Patil, S. L.; Khataniar, S.; Dandekar, A. Y.; Sonwalkar, V. S. Evaluation of electromagnetic heating for heavy oil recovery from Alaskan reservoirs. SPE-154123-MS. SPE Western Regional Meeting, Bakersfield, California, USA, 21-23 March, 2012.

https://doi.org/10.2118/154123-MS

Ruiz C. P. A.; Tarifa J. M.; Gomes V. L. A.; Araújo J. S. Influence of cracking reactions during electromagnetic heating. Petroleum Science and Technology, v. 34, p. 940-946, 2016.

https://doi.org/10.1080/10916466.2016.1170847

Sahni, A.; Kumar, M.; Knapp, R. B. Electromagnetic heating methods for heavy oil reservoirs. SPE-62550-MS. SPE/AAPG Western Regional Meeting, Long Beach, California, USA. 1922 June, 2000. https://doi.org/10.2118/62550-MS

Tarifa J. M.; Ruiz C. P. A.; Gomes V. L. A.; Araújo J. S. Coke deposition during electromagnetic heating. Petroleum Science and Technology, v. 35, p. 1-8, 2017.

https://doi.org/10.1080/10916466.2016.1243129 